Potential aber noch längst nicht dahingehend genutzt wird, dass von einem $\mathrm{Pa}$ radigmenwechsel gesprochen werden könnte. Das liegt zum einen daran, dass auch digitale Projekte konzeptionell weiterhin am klassischen Medium Buch orientiert sind und bloß traditionelle Ansätze zu übertragen versuchen; zum anderen fehlen bisher etablierte Standards, wie kirchenhistorische Daten allgemein $\mathrm{zu}$ identifizieren sind, so dass ein projektübergreifender Datenpool entstehen könnte. Eine Umstellung von projektspezifischen Daten-Idiosynkrasien hin zu einer standardisierten Semantisierung könnte sich aber gerade für kirchengeschichtliche Projekte als bahnbrechend erweisen, weil deren Gegenstände - ob es sich nun um Text-, Personen- oder Objektdaten handelt - meist in einem historischem Beziehungsgeflecht stehen, das komplexer ist, als es ein einzelnes Projekt abbilden kann. Hier sind allerdings die Kirchenhistorikerinnen und -historiker als Community gefragt: Weil erstens das Datennetz keine Grenzen und keine Obrigkeiten kennt, wird sich eine gemeinsame Semantik nur durch deren tatsächliche Nutzung, Überprüfung und Verfeinerung in konkreten Projekten mehr und mehr etablieren können. Zweitens kann sich ein solch allgemeiner Datenpool nur aus Projekten speisen, die einen klaren Open data-Ansatz verfolgen, so dass ein automatisierter Datenaustausch überhaupt möglich wird. Ist die Community bereit, sich auf diese Herausforderungen einzulassen, dann sind eine Vielzahl an neuen Zugängen zu kirchengeschichtlichen Daten und damit an neuen Fragen und schließlich auch an neuartigen Erkenntnissen möglich.

\title{
»Das Internet ist für uns alle Neuland « Zum Verbältnis der Enzyklopädie Wikipedia zur theologisch-ethischen Wissenschaft
}

Reiner Anselm und Lukas David Meyer

Peter Dabrock, Bioethik des Menschen, in: Wolfgang Huber/Torsten Meireis/Hans-Richard Reuter (Hg.), Handbuch der Evangelischen Ethik, C.H. Beck München 2015, 517-583. - Ulrich Duchrow/Wolfgang Huber (Hg.), Die Ambivalenz der Zweireichelehre in lutherischen Kirchen des zwanzigsten Jahrhunderts (TKTG 22), Gütersloher Verlagshaus Mohn Gütersloh 1976, 242 S. - Wikipedia. Die freie Enzyklopädie, Art. Zwei-Reiche-Lehre, online: https://de.wikipedia. org/wiki/Zwei-Reiche-Lehre (12.06.2020). - Wikipedia. Die freie Enzyklopädie, Art. Bioethik, online: https://de.wikipedia.org/wiki/Bioethik (12.06.2020).

\section{Weitere Literatur}

Reiner Anselm, Politische Ethik, in: Wolfgang Huber/Torsten Meireis/Hans-Richard Reuter (Hg.), Handbuch der Evangelischen Ethik, C.H. Beck München 2015, 195-263. - Hans Michael Heinig (Hg.), Aneignung des Gegebenen. Entstehung und Wirkung der Demokratie-Denkschrift der EKD, Mohr Siebeck Tübingen 2017, XIV + 126 S. - Arnd Henze, Kann Kirche 
Demokratie? Wir Protestanten im Stresstest, Herder Freiburg 2018, 175 S. - Kurt Nowak, Zweireichelehre. Anmerkungen zum Entstehungsprozess einer umstrittenen Begriffsprägung und kontroversen Lehre, in: ZThK 78 (1981) 105-127. - Uwe Siemon-Netto, Luther als Wegbreiter Hitlers? Zur Geschichte eines Vorurteils. Mit einer Einführung von Peter L. Berger, Gütersloher Verlagshaus Gütersloh 1993, 150 S.

\section{Einleitung}

Im Juni 2013 verbreitete sich eine Aussage der Bundeskanzlerin Angela Merkel rasch auf Twitter: »Das Internet ist für uns alle Neuland.« Für das nur schwer zu stillende Bedürfnis der Community nach Empörung und Sarkasmus war das ein gefundenes Fressen: Wie so häufig dekontextualisierte sie ein Zitat, um in diesem Fall Merkel zu verspotten. So komisch der Satz für digital natives auf den ersten Blick erscheinen mag, so berechtigt war er hinsichtlich seiner eigentlich geopolitischen Fragestellung: Denn die Bundeskanzlerin hatte das Neuland nicht etwa durch eine verspätete Umstellung von Brief auf E-Mail betreten, sondern es auf das von der NSA geführte Programm PRISM bezogen. Mit Blick auf einen stabilen internationalen Rechtsrahmen war und ist das Internet also in der Tat Neuland - selbst für engagierte Twitter-User.

Die unterschiedlichen Perspektiven, die hier zwischen digital natives und digital migrants zu beobachten sind, lassen sich ohne Weiteres auch für den Umgang mit dem Internet in der theologischen Ethik beschreiben. So wird insbesondere in der universitären Lehre deutlich, dass Studierende vor allem digitale Quellen nutzen - gleichzeitig operiert der theologische Wissenschafts- und Publikationsbetrieb aber weitgehend print-orientiert. Dem steht etwa die PISA-Studie von 2018 gegenüber, die wie zahlreiche vorangegangene Studien die Verlagerung des Leseverhaltens in die digitale Sphäre und eine Verringerung der Lesemotivation ausmachte. Müssen also die Studierenden noch intensiver humanistisch nachbeschult werden, am besten mit analogen Quellen? Oder sollten sich die Dozierenden von ihrer antiquierten Bildungssentimentalität lösen und Theologie endlich zeitgemäß und im digitalen Sinne at its best - d.h. konzentrierter, konziser und problemorientierter - vermitteln?

Bei der Beantwortung dieser extrapolierten Fragen lässt sich nicht einfach zwischen der Verfügbarkeit digitaler Quellen und ihrer Nutzung unterscheiden. Digital migrants nutzen das Internet selbstverständlich auch, allerdings primär als Hilfsmedium für den Zugang zu etablierten Quellen. Digital natives dagegen sehen in ihm eine eigenständige Form der Generierung und nicht nur der Distribution von Wissen, die neben, möglicherweise sogar an Stelle der etablierten Formen treten soll. Mit dieser Differenz geht, so die These unseres Beitrags, eine Verschiebung der Verifikationsinstanzen einher. Sie stellt gerade die evangelische Theologie, besonders aber die von vornherein pluralistisch konzipierte Ethik vor Herausforderungen und ist möglicherweise verantwortlich für die Skepsis, die in der Wissenschaft den neuen Quellen entgegengebracht wird: Denn an die Stelle 
der Autorisierung durch eine identifizierbare (Forscher-)Persönlichkeit tritt die Autorisierung durch eine nicht näher fassbare Community. Besonders deutlich wird dies im Fall von Wikipedia, die im Fokus dieser vergleichenden Analyse steht. Natürlich ist dabei die Kontrastierung zwischen diesen beiden Autorisierungsarten idealtypisch, neben Wikipedia als dem meistverbreiteten Beispiel für community intelligence gibt es auch zahlreiche Mischformen, etwa dort, wo das Prinzip etablierter Enzyklopädien in den digitalen Raum übertragen wird. Die Stanford Encyclopedia of Philosophy bildet hier ein ebenso einschlägiges Beispiel wie der »Brockhaus«.

\section{Vergleichende Fallbeispiele: Handbuch der Evangelischen Ethik (HEE) versus Wikipedia}

Um diese These zu erläutern, gehen wir zunächst komparativ vor: Hierbei wollen wir theologisch-ethische Debatten mit ihrer Darstellung in der deutschsprachigen Online-Enzyklopädie Wikipedia abgleichen. Zwei unterschiedlich gelagerte Fallbeispiele, einmal, aus dem Bereich der politischen Ethik, die Perspektivierung der Zwei-Reiche-Lehre (ZRL), einmal der Umgang mit den Grundlagen- und den Anwendungsfragen der Bioethik, sollen verdeutlichen, zu welchen Konsequenzen die Ausrichtung an den unterschiedlichen Paradigmen führt. Dabei wird sich zeigen, dass die Zeitgemäßheit des Mediums keineswegs notwendig einhergehen muss mit Ergebnissen der aktuellsten Forschung. Diskussionswürdig scheinen uns abschließend besonders zwei Fragen: Welche Umgangsweise mit Verifikationsinstanzen ist angemessen? Und wie ist das Verhältnis zwischen einer wissenschaftlichen und einer popularisierenden Öffentlichkeit genauer zu bestimmen? In beiden Bereichen gibt es deutliche Desiderate, die zu bearbeiten uns selbst eine Frage der Ethik zu sein scheint.

Dabei soll keineswegs in entlarvender Manier offengelegt werden, dass und inwiefern das etablierte wissenschaftliche Reflexionsniveau für die auf Wikipedia aktiven Prosumer unerreichbar sei. Vielmehr soll, in Anlehnung an eine systemtheoretische Perspektive, die Eigenständigkeit der jeweiligen Logiken anerkannt werden, um die Interferenzen genauer zu bestimmen. Wikipedia ist dabei als populärwissenschaftliches Leitmedium zu würdigen. Qualitative Aspekte spielen dabei noch keine Rolle, der Blick auf die Zahlen genügt: Im deutschsprachigen Raum nutzen einer Statista-Studie zufolge vier von fünf Internetusern regelmäßig Wikipedia zur Informationsbeschaffung, bei der Generation zwischen 14 und 29 Jahren sind es sogar 92 Prozent.

\section{a) Politisch-ethisches Fallbeispiel: die Zwei-Reiche-Lehre als Innerlichkeits- oder Entsäkularisierungsproblem?}

Die Debatte um die Zwei-Reiche-Lehre (ZRL) kann als einer der wichtigsten Stränge der politisch-ethischen Selbstverständigung der evangelischen Staatslehre nach 1945 gelten. Über die Notwendigkeit eines Neuanfangs nach dem Zweiten Weltkrieg bestand ein breiter Konsens - strittig war indes, wie dieser erfolgen 
sollte. Entscheidend dafür war die Problemdiagnose hinsichtlich der lutherischen ZRL. Führte sie zu einer privatisierenden Innerlichkeit, die das Politische in eine säkulare >Eigengesetzlichkeit< entlassen und durch einen theologischen Quietismus wehrlos gegenüber dem NS-Regime gemacht hatte? Oder war nicht die Innerlichkeit, sondern gerade umgekehrt die lutherische Sakralisierung des Staates als >gute Regierweise < und >gottgewollte Obrigkeit< das zentrale Problem, da so der NS-Staat metaphysisch überhöht werden konnte?

Mit diesen entgegengesetzten Alternativen war ein unterschiedliches Verhältnis zur politischen und gesellschaftlichen Ordnung der Bundesrepublik verbunden: »Theologie der Gesellschaft«, also »Aneignung des Gegebenen« (H.-M. Heinig) oder »Theologische Sozialethik «, also Profilierung als kritisches Gegenüber zum politischen Umfeld. Dass sich diese Differenz zudem mit unterschiedlichen politischen Präferenzen verband, verlieh der Kontroverse zusätzliche Schärfe, verhalf ihr aber auch zu einer recht hohen Aufmerksamkeit in den politischen Orientierungsdebatten der Bonner Republik. Der Dreiklang Luther-Bismarck-Hitler wurde diskreditierend gegen eine Affinität zum Gegebenen als antidemokratische Grundüberzeugung angeführt; umgekehrt wollten Gegner einer Theologischen Sozialethik diese als moralisierend und realitätsfremd ins Abseits stellen. Die intensiv geführte Debatte verdichtete sich in den späten 1970er und frühen 1980er Jahren, als U. Duchrow und W. Huber für die Innerlichkeitsdiagnose argumentierten (Duchrow/Huber), während M. Honecker oder T. Rendtorff im Gegenzug diese Interpretation als »Konstrukt der neuesten Theologiegeschichte « verwarfen. Erst mit der Demokratiedenkschrift von 1985 gelang es, die verfestigten Fronten zu überwinden: Trotz offenkundiger Divergenzen kamen auch die beiden großen Antipoden - Rendtorff auf der einen, Huber auf der anderen Seite - zu einem differenzierten Konsens (vgl. Heinig). In materialen politischen Streitfragen entluden sich allerdings die Spannungen erneut, sodass sich die Frontstellung zwischen linksprotestantischer Positionsfreudigkeit und variantenreicher lutherischer Zurückhaltung wiederholte.

Im HEE wird versucht, diese Debatte historisch zu kontextualisieren und im Einklang mit der neueren theologiegeschichtlichen Forschung darauf hinzuweisen, dass beide Positionen sich im Grunde näher standen, als es die unterschiedlichen politischen Positionen der Nachkriegszeit und die damit verbundenen konkreten Parteinahmen vermuten ließen: Es ist die reformatorische, besonders die lutherische Staatsmetaphysik, die zu einer steten theologischen Überlegitimierung der eigenen Einschätzungen neigte. Erst die mit der Demokratiedenkschrift begonnene Abkehr von diesem Denken eröffnete neue politisch-ethische Spielräume; insbesondere die Figur der Entsprechungen bzw. der Ähnlichkeiten führte zur Suche nach Parallelen zwischen christlich-protestantischen Überzeugungen und dem freiheitlich-demokratischen Rechtsstaat, ohne auf einem theologischen Exklusivitätsanspruch zu beharren. Das bedeutet zugleich, dass nachfolgende politische Auseinandersetzungen so kontrovers sind, weil die konkreten Einschätzungen und Zielsetzungen im Bereich des Politischen ebenso umstritten sind wie 
eine entscheidungsbezogene Analogiebildung zu entsprechenden grundlegenden Überzeugungen des Christentums. In dieser Perspektive will also die Darstellung der ZRL im HEE auf die Kontroversen der Nachkriegszeit zurückblicken, diese historisieren und einer Transformation im christlichen Glauben zuführen, die den Abstand zwischen der Sphäre des Politischen und der Sphäre des Absoluten wahrt. Auch wenn im Sinne der hier kurz wiedergegebenen Deutung durchaus konstatiert werden kann, dass die Schärfe der theologisch-ethischen Kontroversen deutlich nachgelassen hat, so wirken alte Frontstellung weiter. Bereits der Leipziger Kirchenhistoriker K. Nowak bemerkte während der Debatte der 1980erJahre, dass die Vereinseitigung des Luthertums als politisch-passiv und letztlich demokratieunfähig bereits bei E. Troeltsch, K. Barth und D. Bonhoeffer virulent war und im »Laien-Schrifttum « weiter tradiert werde (Nowak, 126). Diese Beobachtung lässt sich auch in der >Laien-Enzyklopädie< Wikipedia (Art. Zwei-Reiche-Lehre) bestätigen. Der additiv strukturierte Artikel betont die Kontextbezogenheit der Lutherschen Aussagen sowie die erst im 20. Jh. einsetzende Systematisierung zu einer klarer konzipierten Lehre. Bemerkenswert ist, dass die von Nowak bereits vor 40 Jahren angestellte Diagnose sich belegen lässt. So heißt es hier: Bereits E. Troeltsch beobachte einen durch die ZRL vermittelten »Rückzug der Christen in den Privatbereich und so die Überlassung der Politik in ihrer vermeintlichen Eigengesetzlichkeit«. Daraus entspringe, wie erneut Troeltsch in den Mund gelegt wird, eine Deutung des Staates als von Gott vorgegebene Ordnung, »ob Diktatur, Monarchie oder Republik, ihr kommt unbedingter Gehorsam zu«. Troeltsch schrieb dem Luthertum tatsächlich die Innerlichkeit und damit die Affinität zur Monarchie zu; dem Reformiertentum attestierte er hingegen über die Betonung der Freiheitsrechte eine maßgebliche Bedeutung bei der Entstehung republikanischer Ideen. In der Wikipedia-Darstellung wird die Innerlichkeitsthese allerdings weniger mit Demokratieunfähigkeit, als vielmehr mit genereller politischer Devotion verknüpft. Damit nicht genug der Großthesen: K. Barth wird zugeschrieben, dass er »nicht ohne Grund [...] eine historische Linie von Luther über Friedrich den Großen und Bismarck zu Adolf Hitler ziehen und damit die ZRL kritisieren« konnte.

Zwei Textstellen scheinen hier im Hintergrund zu stehen, ohne explizit genannt zu werden: »Konsequenter konnte das Werk Friedrichs des Großen und Bismarcks nicht vollendet, und gründlicher konnte es nicht zerstört werden als es durch Adolf Hitler geschehen ist « (K. Barth, Eine Schweizer Stimme. 1938-1945, Zollikon-Zürich 1945, 346). Außerdem: »Die Linie Friedrich-Bismarck-Wilhelm II-Hitler wird immer und immer wieder gezogen. Preußen ist zum roten Tuch geworden« (a.a.O., 386).

Obwohl U. Siemon-Netto ein - aus dem Duktus allerdings herausfallender Absatz im Artikel gewidmet ist, wird dessen kritische Aufarbeitung dieser These nicht erwähnt (Siemon-Netto). Die ZRL, so würde der Leser wohl an dieser Stelle annehmen, leistet also vieles, aber gewiss keine Anerkennung der modernen, freiheitlichen Demokratie. Allerdings folgt dann eine interessante Wendung: 
Die Theologie der Ordnungen und das Konzept der Königsherrschaft Christi werden beide als Ausdrucksformen der ZRL gedeutet, die in der Barmer Theologischen Erklärung konvergieren und auch nur zusammen eine sachgerechte politische Ethik des Protestantismus ergeben könnten. Ehe sich die Darstellung in argumentativen - und auch sprachlichen - Untiefen verirrt, flüchtet sich der Artikel wieder in das, was ohne Zweifel die Stärke von Wikipedia darstellt: kurze Faktendarstellung. Unterschiedliche Interpretationsansätze werden durchaus dar-, allerdings ohne weiteren Kommentar nebeneinandergestellt.

Wissenschaftlich Geschulte mögen nun belustigt oder empört reagieren. Hierbei ist zunächst auf den notwendig provisorischen Charakter von Wikipedia-Artikeln sowie die Enzyklopädie-eigenen Prüfmechanismen hinzuweisen. Ein ,lesenswerter Artikel' setzt klare Kriterien voraus - fachliche Korrektheit, zugängliche Form und Sprache, angemessene Belege -, diese sind nach Maßstäben der Enzyklopädie nicht erfüllt, entsprechend bürgt auch kein Siegel für Qualität. Gleichwohl unterbietet der Artikel auch die eigentlich vorhandenen Prüfmechanismen, da keine Infragestellung der gegebenen Belege sichtbar ist. Unter Absehung der mangelnden Präzision lässt sich aber durchaus festhalten, dass der Wikipedia-Artikel im Grundzug der von Duchrow und Huber Ende der 1970erJahre formulierten Kritik an der ZRL folgt, wenn er mit beiden Gewährsleuten sowie H.-E. Tödt und der entsprechenden Arbeitsgruppe des Kirchenbundes der DDR eine Interpretation der ZRL von Barmen II her vorschlägt: »So wird dem Gebot Gottes eine korrigierende und legitimierende Kraft gegenüber der natürlichen Vernunft zugesprochen. « Diese Positionierung mag auch mit der Eingängigkeit der These zusammenhängen, die durch eine zwar undifferenzierte, aber klare Bestimmung des Luthertums eine Attraktivität aus der eindeutigen Zuordnung bezieht. Das Kerninteresse scheint dabei eher beim Wunsch nach klarer kirchlicher Profilierung in gegenwartspolitischen Fragen zu liegen, zu dem dann eine ungenau rekonstruierte großgeschichtliche These ergänzend hinzutritt. Aus einem zunächst diffusen Gefühl, der Protestantismus sei angesichts einer drängenden Gegenwartsfrage zu passiv, gewinnt die Innerlichkeits-These offenbar an Plausibilität, wie die - allerdings eher populärwissenschaftliche - Studie von A. Henze zeigt (Henze).

Die wissenschaftliche und die popularisierende-mediale Öffentlichkeit stehen, wie der Vergleich zeigte, ziemlich disparat nebeneinander. Der Versuch, eine eigenständige Zusammenschau der Perspektiven zu bringen, wird in Wikipedia eigentlich nicht unternommen, es dominiert die Aneinanderreihung von Fakten, über die, offenbar im Interesse der Stärkung der allerdings nur abschließend in einem Bonhoeffer-Zitat erwähnten Figur eines Wächteramts, eine nicht weiter hergeleitete oder vertretene Interpretation gelegt wird. 


\section{b) Bioethisches Fallbeispiel: wann wird das Ganze, wann das Spezifische thematisch?}

Bei der Auseinandersetzung mit einem bioethischen Fallbeispiel bietet sich eine generelle Auseinandersetzung mit dem Status theologischer Grundorientierungen in bioethischen Debatten an. Anders als im politisch-ethischen Bereich macht der technische Fortschritt eine intensive Auseinandersetzung mit neu aufgeworfenen Detailfragen erforderlich. Allerdings wäre es ein Missverständnis, die ethische Reflexion auf die Klärung spezifischer Detailfragen zu beschränken, da die Wahrnehmung des Spezifischen stets durch eine rahmende Grundorientierung beeinflusst wird. Insofern ist die theologische Ethik - um eine Formulierung H. Lübbes aufzunehmen - angesichts bioethischer Probleme nicht für alles, aber für das Ganze zuständig - sie kann Grundorientierungen reflektieren, sollte Detailfragen kennen, sich aber nicht anmaßen, diese dann auch noch zu klären. Diesem Grundgedanken korrespondiert der Aufbau des Beitrags von P. Dabrock im Handbuch für Evangelische Ethik. Als Grundorientierung kommt die ZRL neben Gesetz und Evangelium als eines von zwei zentralen Theologoumena zur Sprache:

»Deren theologisch-ethische Funktion, auf die Gott-Mensch-Differenz hinzuweisen, und zwar vor allem in soteriologischer Absicht, lässt sich auch in außertheologischen Diskursen als Grenzmarker gegen allzu forsche biotechnologische Heilsversprechen zur Geltung bringen. Dazu müssen sie freilich in einem formaleren Ansatz eingebunden werden, der nicht auf die Inhalte einer bestimmten Religion festgelegt ist, partikulare Traditionen aber trotzdem würdigen kann«(525).

In der notwendigen Ausmittlung von Universalität und Partikularität wird die Unterscheidung zwischen weltlicher und geistlicher Sphäre relevant: angesichts der Unbedingtheitsdimensionen bioethischer Probleme gilt es, die Bedingtheit pluraler Grundorientierungen bei der Wahrnehmung dieser Probleme in Erinnerung zu rufen. Fragen der Lebensführung verlangen selten Einstimmigkeit, während im schlimmsten Fall ein status confessionis eine spezifische Problemwahrnehmung mit einem generellen Heilsanspruch verwechselt. Die ausdrückliche Würdigung von Pluralität ist nicht mit resignativer Beliebigkeit zu verwechseln. Denn auch im Bewusstsein der eigenen Partikularität vermag eine evangelisch-theologische Bioethik es, anthropologische Grundorientierungen zum Ausdruck zu bringen, etwa die als Gottebenbildlichkeit interpretierte Würde der Person, die rechtfertigungstheologisch konturierte Einsicht in ihre Fragilität, oder die durch kommunikative Freiheit bedingte Angewiesenheit auf die Nächsten. Dabei ist nur wichtig, »den erwartbaren Dissens in biopolitischen und -ethischen Deliberationsprozessen zumindest in gut ausgehandelte, auf Rechtsfrieden zielende Kompromisse « (536) zu lenken. Theologische Bioethik sollte also im changierenden Wortsinn ,selbst-bewusst' sein: sie soll ihre Grundorientierung als solche bestimmen, sie engagiert ins Gespräch bringen und für Kompromisse offenbleiben. 
Inwiefern wird nun in der Online-Enzyklopädie das Ganze und das Spezifische thematisch? Grundsätzlich lässt sich festhalten, dass die Stärken vor allem in der Darstellung spezifischer Sachverhalte liegen. Die stetigen Aktualisierungen unter teils hitzigen Diskussionen einer oft detailverliebten Autorenschaft ermöglichen es, technische Fortschritte und mitunter schnelle Rechtsentwicklungen abzubilden. Entsprechend würdigt die Community vor allem Artikel zu spezifischen Ethikbereichen als >lesenswert< oder teils gar als >exzellent<, während der Artikel zum Ganzen der Bioethik keine solche Anerkennung erfährt - anders übrigens als der noch allgemeinere Artikel zum Thema 'Ethik ‘. Indes weist die Gliederung des Wikipedia-Artikels zur Bioethik eine gewisse Ähnlichkeit zum vorab rekapitulierten Handbuch-Artikel Dabrocks auf - einer begrifflichen Einleitung folgt ein prinzipieller Abschnitt zu »bioethischen Herangehensweise«, ehe abschließend auch konkrete Problemfelder exemplifiziert werden. Als Grundorientierungen sind zwei Cluster aufgeführt: einerseits wird ein deontologischer einem utilitaristischen Ansatz, andererseits ein liberaler, an Individualrechten interessierter Ausgangspunkt einem kommunitaristischen, auf die gesamtgesellschaftlichen Auswirkungen bedachter Ansatz gegenübergestellt. Die Beschränkung auf philosophisch-ethische Grundorientierungen dürfte erklären, warum keine gesonderte Reflexion universaler Geltungsansprüche im Verhältnis zur Partikularität der Konfliktparteien sowie die Suche nach Kompromissen stattfindet.

Der Artikel im Besonderen weist somit einen Aspekt auf, der bei Durchsicht weiterer bioethischer Artikel verallgemeinert werden kann: Bioethische Fragen werden vor allem technisch-rechtlich und eher unter Rückgriff auf philosophische Überlegungen verhandelt, weder kirchliche noch theologische Positionierungen nehmen einen der politischen Öffentlichkeit in Deutschland vergleichbaren Raum ein. Ethische Grundorientierungen stehen zudem meist additiv nebeneinander.

\section{Bilanz und Desiderat}

Die Auseinandersetzung mit zwei Fallbeispielen hat gezeigt, dass die schwach ausgeprägten Interferenzen zwischen Wissenschaftsbetrieb und der digitalen Öffentlichkeit in ein konstruktiveres Verhältnis zueinander gesetzt werden können. Von theologischer Seite aus setzt das einerseits die Kenntnis der Plattform-eigenen Mechanismen, aber auch die Würdigung ihrer Bedeutung voraus. In der heutigen Generation der Studierenden nutzen mehr als neun von zehn Personen regelmäßig Wikipedia, das als der zentrale positive Wissensbestand der Gegenwart gelten kann. Diese Einschätzung ist indes nicht positivistisch misszuverstehen: Denn die Darstellung ethischer Probleme ist nie einfach $>n e u-$ tral<, wie mitunter User auf der Plattform oder analytisch-philosophische Traditionen insinuieren. Die theologische Ethik sollte das durch die stete externe Hinterfragung ihres Universalitätsanspruchs produktive Reflexionspotenzial 
stärker einbringen. Ein solcher Beitrag beschränkt sich dabei nicht allein auf den textbasierten digitalen Raum, sondern schließt auch visuelle Darstellungsformen ein. Es impliziert eine konzeptuelle Anpassung an die digitale Sphäre und nicht das bloße Abfilmen eines Frontalvortrages - so brillant sich der Vortragende auch finden mag. Insofern beinhaltet die Verfolgung dieses Desiderats erhebliche Übersetzungsanstrengungen, dürfte aber vor allem im Rahmen der Lehre ein wichtiger akademischer Dienst der Nächstenliebe sein. So nachvollziehbar die Lust an humanistischer Empörung über die Jugend ist, so unproduktiv ist es, nicht an ihr Nutzungsverhalten anzuknüpfen.

Wie bereits angedeutet, würde es aber entschieden zu kurz greifen, diese Übersetzung als bloße Übertragung der guten alten Quellen in die schlechte neue Online-Welt zu verstehen. Die Dinge liegen deutlich komplizierter: Denn der Vergleich zwischen den beiden Themenbereichen offenbart ja auch, dass im Blick auf die reine Faktendarstellung Wikipedia traditionellen Print-Medien oder auch traditionellen Online-Enzyklopädien nicht nachsteht, im Gegenteil. Qualitätsmerkmal von Wikipedia sind die hohe Aktualität, in der Regel auch die hohe Detailaffinität bei der Analyse der materialethischen Themen. Demgegenüber tritt die abwägende Reflexion und auch die begründete ethische Argumentation eher in den Hintergrund, hier sind die traditionellen Quellen im Vorteil. Diese unterschiedlichen Stärken dürften weniger in der Qualifikation der Autoren als vielmehr in dem zugrunde gelegten Wissenschaftsverständnis liegen.

Denn im Hintergrund von Wikipedia steht der Gedanke, dass wissenschaftliche Wahrheit durch eine demokratisch legitimierte crowd intelligence erreicht bzw. dargestellt werden kann. Das führt dazu, dass der Stil der additiven Aneinanderreihung von objektivierbaren Faktoren dominiert, der Darstellung von Fakten und Zusammenfassung von Positionen. Die Interpretation der Fakten steht demgegenüber deutlich zurück; Positionalität ist in einem Medium, das sehr auf Konsensfindung aufbaut, schwierig. Hier erschwert auch die anonymisierte oder digital entzogene Autorschaft eine Identifizierung der einzelnen Positionen und damit einen direkten Diskurs. Das allerdings verstärkt das Bestreben, sich an vermeintlichen Objektivitäten zu orientieren. Bei den materialen Fragen der Bioethik gelingt dies deutlich besser als bei der Rekonstruktion von Theoriefiguren. Für die evangelische Theologie ist diese Beobachtung deswegen eine Herausforderung, weil sie sich, zumindest in den systematischen Fächern, als Interpretationswissenschaft versteht, deren Faktizität stets auch an die Perspektive des Betrachters gebunden ist. Demgegenüber neigt die Wissensaufbereitung bei Wikipedia zu einem Positivismus, der eher zu einer natur- denn zu einem kulturwissenschaftlichen Wissenschaftsverständnis passt. Die Kultur der Digitalität macht somit nicht allein didaktische Neuansätze, sondern auch die wissenschaftstheoretische Auseinandersetzung mit digitalen Phänomenen erforderlich: Wer ein Neuland nicht nur betreten, sondern auch erkunden will, ist auf die Vorarbeit einer orientierenden Kartographie angewiesen. 